\title{
Pilot Study of Industry Perspective on Requirement Engineering Education: Measurement of Rasch Analysis
}

\author{
NOR AZLIANA AKMAL JAMALUDIN \\ Faculty of Computer Science and Information System \\ Universiti Selangor \\ 45600 Bestari Jaya, Selangor \\ MALAYSIA
}

\begin{abstract}
Software development industry identifies that human-based give a significant problem in Requirement Engineering. To that reason, education gives a substantial impact in delivering a skill worker and should be a medium to reduce the problem. Survey question was distributed among ICT for this pilot study to the organization of MSC status in Malaysia for pilot study. $15.53 \%(N=32)$ respondent successfully return their respond back. The result shows that only 27 person is analyzed regarding to misfit data provided by Rasch Measurement Model. The unidimensionality, person-item map and misfit data are discussed. Research objective to identify the undergraduate problem in Requirement Engineering education is achieved. Future work will be discussed on further analysis on actual survey to improve employability skill among software engineering undergraduate students.
\end{abstract}

Keywords-Higher Learning Education; Requirement Engineering; Education; Rasch Measurement Model; Employability Skill; Undergraduate Problem; Rasch Analysis; Unidimensionality; Human-based Problem.

\section{INTRODUCTION}

Requirement Engineering is a fundamental process to meet stakeholder's needs in software development project. Failure in meeting stakeholder satisfaction will contribute to delay software development project, waste of time, energy, resources and poor quality [22]. The argument of this matter was widely discussed relying on the industry perspective. Industry was spent lots of money in research and development to identify the problem occurs in establishing a strong rapport within stakeholder.

Human-based give a weighty problem to the Requirement engineering. The classification of requirements problems include 1) lack of customer, user and developer 2) lack of communication 3) lack of training 4) lack of define responsibility 5) unstable workforce (low staff retention) 6) inappropriate skills 7) poor time and resource allocations [22].

To that reason, education should be a medium to reduce the human-based problem.

\section{BACKGROUND}

Requirement engineering education can be a weapon to sharpen the human-based skill particularly. Five evidence shows that Prophet Muhammad S.A.W. is an educator that

\author{
SHAMSUL SAHIBUDDIN \\ Advanced Informatics School (AIS), UTM International \\ Campus, Universiti Teknologi Malaysia (UTM) \\ Jalan Semarak, 54100 Kuala Lumpur \\ MALAYSIA
}

takes into account individual differences in delivering teaching that consist of [1]: 1) provide appropriate advice in accordance with the difference that each seeking some advice 2) give a different answer to the same question that tailored to the individual who asked 3 ) behave and be different accordingly to the suitability of the mix therewith 4) deliver and legal adapted to the ability for the person to receive it and 5) implement and receive behavior but a person nor receive from someone else because of the different situations.

Each Higher Learning Education (HLE) has set objectives differently. Towards CGPA or student result, it goes the same meaning for all HLE. Cumulative Grade Point Average or CGPA is used widely in a developing country. Many people assuming that the highest CGPA student will have the highest performance to show their competency $[9,10,11]$.

However, the validity of Cumulative Grade Point Average (CGPA) is purely the mean of raw scores, lack precision and linearity [8] to meet criteria for measure human-based skill should be revised back. The objective of this paper is to identify the problem in the current Requirement Engineering education practice using Rasch Measurement Model.

Figure 1 depicts the current practice process in Higher Learning Education (HLE) that give an input to the current problem arose in HLE. This practice will further analyze in Figure 7.

\section{METHODOLOGY}

The survey was administered randomly from 2201 ICT Malaysia Status Company (MSC) that registered under Multimedia Development Corporation (MDeC). The companies categorized from Shared Service Outsourcing, InfoTech, Creative Multimedia and IHL \& Incubator industry [MDEC, 2012]. Klang Valley is chosen as a location for this pilot study because of an easy accessible respondent and majority MSC organization is located at Klang Valley.

The main objective of this pilot study is to identify the correctness of the questionnaire. Somehow, the questionnaire is expected to give a similar meaning (consistency) to all respondent. If not, some of the questionnaire will be rephrased or removed based on the analysis after data collection in this pilot study. To that reason, consistency in response will be analyzed. In addition, pilot test is used to know an expected 
outcome based on difficult and easy task respond from the respondent.

All observations begin as counts. But raw counts are only indications of a possible measure. Raw counts cannot be the measures sought because in their raw state, they have little inferential value. To develop metric meaning, the counts must be incorporated into a stochastic process which constructs inferential stability.

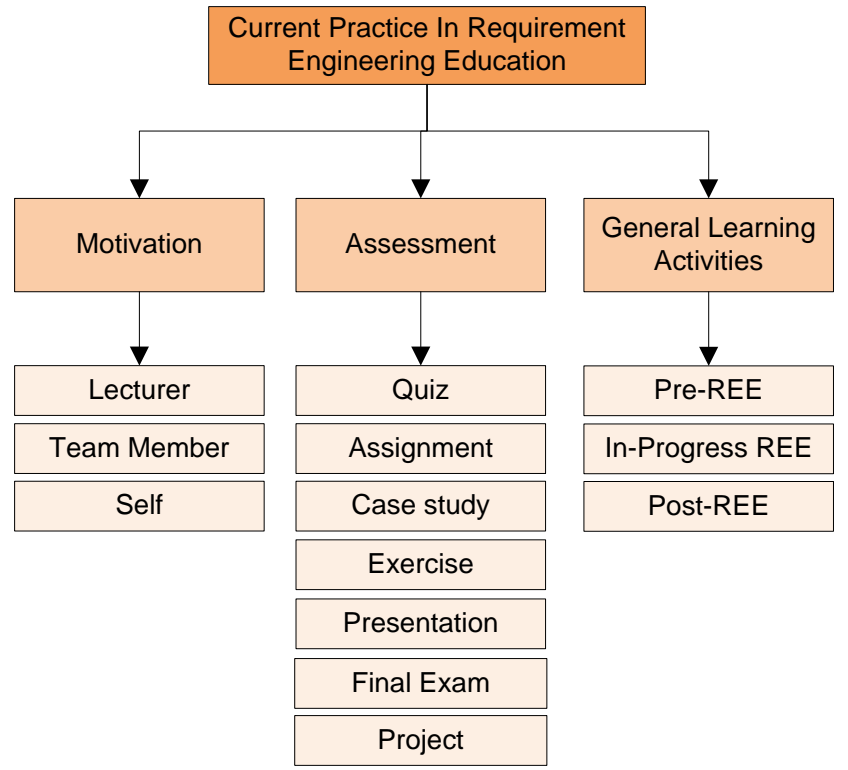

Fig. 1. Process of Current Practice in HLE

Thus, in order to construct inference from observation, the measurement model must: (a) produce linear measures, (b) overcome missing data, (c) give estimates of precision, (d) have devices for detecting misfit, and (e) the parameters of the object being measured and of the measurement instrument must be separable. Only the Rasch measurement models solve these problems. Rasch provide empirical evidence.

\section{VALIDITY OF INSTRUMENT}

The questionnaire is revision based on the Cognitive Domain [4], Bloom's Revised Taxanomy [17] for undergraduate practice [10]. Content $[7,12]$, construct [3] and predictive [13] validity is crucial in getting the sufficient result of the study. Survey questionnaire in this research used two types of scale. First, the dichotomous scale [18] that comprise of $1-2$ scale for 'Yes' and 'No'. The scale is based on management style of decision making. Second, Likert [23] scale $(1-2-3-4-5)$ used for descriptive response categories (never - rarely - sometime - often - always) as a means of partitioning the underlying latent quantitative continuum into successively increasing (or decreasing) amounts of the variable [2].

It leads wrong contextualize the result if we only using ordinal data to achieve the sufficient result. As a solution, a reliable instrument is needed. Rasch shows differently. Rasch transform an ordinal data which is qualitative data into ratio data. Rasch Analysis is deployed vigorously. It used to achieve an effective instrument construct of precision. Rasch able to sieve the instrument clean from any item misfit hence potential data defects $[19,20]$.

\section{Rasch Measurement Model}

Rasch Model is used to analyze data. Application of the Rasch model through software such as Winstep [14] and other Rasch software provide estimates of person and threshold locations on the latent variable scale. The software also yields indices of item and person fit to show that the requirement of unidimensionality is met.

Rasch answer on how to have the right measurement with valid instrument. Instrument is extremely crucial if involve human life. Based on Linacre (2011), things would change appreciably when you want a thermometer fit for an open heart surgery. Certainly we will need a more precise measurement instrument. Life is at stake, so it is necessary to have the correct instrument in place. Cost is no more the issue; precision and reliability overrides all. So it goes in an instrument construct; the Standard Error of Measurement and Item Reliability matters most that ought to be given priority when it comes to high stake measurement.

\section{A. Rasch Analysis}

The normal solution is to apply the regression approach. It shows the best fit line that inline with the points as best as possible. Then, it can be used to make the required predictions by interpolation or extrapolation [8] as necessary as shown in Figure 2.

$$
\mathrm{y}=\beta 0+\beta 1 \mathrm{~m} \quad \quad \text { Equ .. (1) }
$$

In obtaining the best fit line, there exist differences between the actual point; $y$ and the best line, the predicted point; ý. The difference is referred to as error; e.

$$
\text { yi }- \text { ýi }=\text { ei Equ .. (2) }
$$

By accepting the fact that there is always error involved in the prediction model, the deterministic model of equation: 1) can be transformed into probabilistic model by including the prediction error into the equation; Equ. 3) Rasch moves the concept of reliability from establishing "best fit line" of the data into producing reliable repeatable measurement instrument. Rasch focuses on constructing the measurement instrument rather than fitting the data to suit the measurement model. $\mathrm{y}=\beta 0+\beta 1 \mathrm{~m}+\mathrm{e} \quad$ Equ .. (3)

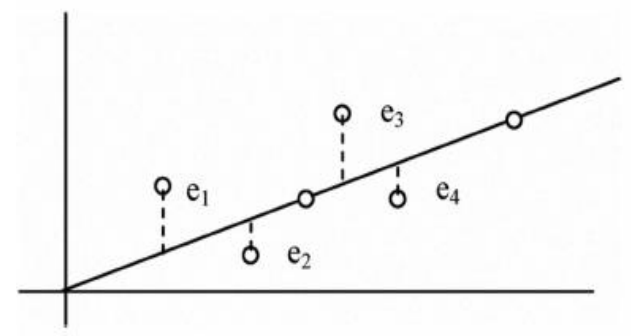

Fig. 2. Best fit line: Linear Regression Model

\section{DISCUSSION}

Only $15.53 \%(\mathrm{~N}=32)$ personnel were successfully returned the survey question. Majority feedback is coming from Shared Service Outsourcing. The services sector is a vital contributor to the growth of the Malaysian economy and 
functions include Information Technology (IT) services, shared services and business process outsourcing (BPO), regional headquarters, research and development (R\&D), training and environmental management.

Malaysia has a vibrant ICT and Services industry that is world-class, confirmed by the AT Kearney 2004 and 2005 Offshore Location Attractiveness Index, which ranks Malaysia as the world's third most attractive Shared Services \& Outsourcing (SSO) location [16]. Rasch help evaluate small sample size that give $95 \%$ confidence level.

\section{A. Summary Statistic}

Result shows in Figure 3 with $84 \%(\mathrm{~N}=27)$ of respondents is a valid response after clean the misfit data. Besides, $77 \%$ of an item is measured after clean the invalid item. A total of 2295 data points arising from 27 respondents on 85 items was analyzed. It yields a Chi-Square value of 3493.40. Cronbach- $\alpha$ value is 0.90 , which contribute high reliability of raw score for the instrument in measuring the undergraduate problem.

The optimal categorization [15] in which provides the best construct definition, best separates respondents along the variable, and produces the best fit of data to model. Targeting is at 0.81 logit $(\mathrm{S} . \mathrm{E} .=0.13)$ which referring to MeanPerson MeanItem (0.81 logit -0.00 logit). Targeting is less than 1 logit. Based on a rating scale instrument quality criteria; if targeting $<1$ error, then it is good targeting. So, the instrument is on target.

In a mean time, it showed a "Good" reliability (Fisher, 2007) for both item and person reliability. The item is sufficient at 0.71 that above from 0.7 (reliability $>0.7$ ) give a meaning that the instrument has acceptable number of items to measure what is supposedly to be measured in the underpinning theory.

\begin{tabular}{|c|c|c|c|c|c|c|c|c|}
\hline & \multirow{2}{*}{$\begin{array}{c}\text { RAW } \\
\text { SCORE }\end{array}$} & \multirow[b]{2}{*}{ COUNT } & \multirow[b]{2}{*}{ MEASURE } & \multirow{2}{*}{$\begin{array}{l}\text { MODEL } \\
\text { ERROR }\end{array}$} & \multicolumn{2}{|c|}{ INFIT } & \multicolumn{2}{|c|}{ OUTFIT } \\
\hline & & & & & MNSQ & ZSTD & MNSQ & ZSTD \\
\hline$M E A N$ & 58.0 & 27.0 & .00 & .37 & 1.00 & .0 & .99 & -.1 \\
\hline S.D. & 18.2 & .0 & .72 & .08 & .18 & .9 & .18 & .8 \\
\hline MAX. & 88.0 & 27.0 & 1.94 & .74 & 1.46 & 1.9 & 1.49 & 1.8 \\
\hline MIN. & 34.0 & 27.0 & -1.88 & .29 & .48 & -2.8 & .49 & -2.6 \\
\hline
\end{tabular}

REAL RMSE .39 ADJ.SD .61 SEPARATION 1.57 Item RELIABILITY . 71 MODEL RMSE.38 ADJ.SD .62 SEPARATION 1.63 Item RELIABILITY .73 S.E. OF Item MEAN $=.08$

Fig. 3. Summary of Measured 85 Items

The person reliability is sufficient at 0.90 indicates a high reliability. This gives an indication that the instrument can differentiate the person ability with the difficult past practices in undergraduate study. Furthermore, the item was sufficient to separate the person; PSI 3.08 logit into four groups, which match the expectation (CGPA categories). Expected person based on CGPA categories is 0.00-1.99 (D), 2.00 - 2.99 (C), $3.00-3.49$ (B) and $3.50-4.00$ (A). No person is in D categories.

MeanItem from Figure 4 is set to an arbitrary 0.00. The instrument where 'zero-setting' all items is at 50:50 situation. Error $0.37(>0.25)$ is slightly high. MeanPerson give a value of +0.81 logit; Person meet expectation. The personitem map in Figure 6, reveals that there is one poor person is located below MeanItem and has low ability with -0.28 logit. The Excellent person is at 2.79 logit, which above the highest located practice at 1.94 difficulty logit. Inspite of the good reliability, more difficult items. However, the items need to be introduced for that gap of 0.95 logit

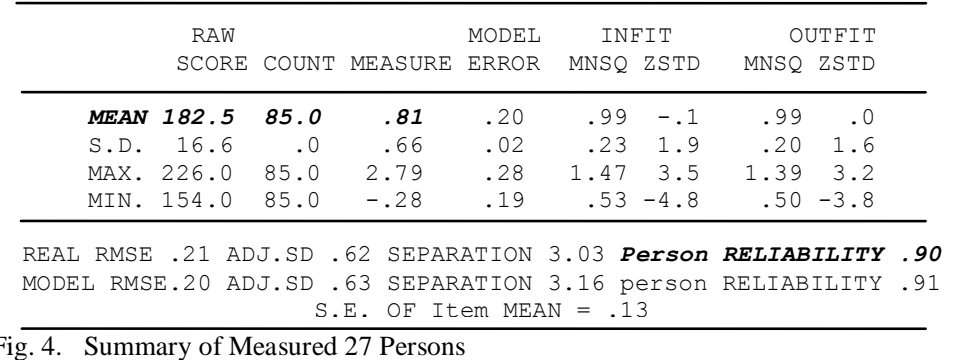

\section{B. Person and Item Fit}

To fit the item into the model, we should first identify the sum of Mean and Standard Deviation (SD) to clean the data based on Point Mean Correlation (PTMEA), Mean Square (MNSQ) and z-standard (ZSTD). If the data is indicated high Z-std that bigger than 1.29 logit, it is person misfit. Figure 5 shows person 20, 27, 31, 32 and 13 are misfit with MNSQ > 1.25 logit and z-std $> \pm 2$ logit. Item whose MNSQ is nearer to 1 and z-std nearer to 0 is deemed a better fit. However, Point of Mean (PTMEA) Correlation allow the negative response because the study is to identify the competence graduates students that get low CGPA but has a high skill. Ignore if in between the range of $0.5<\mathrm{MNSQ}<1.5$ and ZSTD \pm 2 logit.

\begin{tabular}{rrrrrrr|rrr||rr}
\hline ENTRY & TOTAL & & \multicolumn{4}{c|}{ MODEL } & \multicolumn{2}{c|}{ INFIT } & \multicolumn{2}{|c}{ OUTFIT } & \multicolumn{2}{c|}{ PTMEA } & EXACT MATCH \\
NUMBER & SCORE & COUNT & MEASURE & S.E. & MNSQ & ZSTD & MNSQ & ZSTD & CORR. & OBS\% & EXP\% \\
\hline 8 & 226 & 85 & 2.78 & .28 & .72 & -1.3 & .50 & -1.8 & .10 & 83.5 & 82.7 \\
32 & 218 & 85 & 2.26 & .24 & .77 & -1.3 & .56 & -2.1 & -.01 & 80.0 & 74.3 \\
31 & 210 & 85 & 1.86 & .21 & 1.23 & 1.4 & .83 & -.9 & -.05 & 71.8 & 68.0 \\
17 & 209 & 85 & 1.81 & .21 & 1.02 & .2 & .74 & -1.5 & -.16 & 76.5 & 67.7 \\
27 & 209 & 85 & 1.81 & .21 & .42 & -4.8 & .36 & -4.5 & -.32 & 89.4 & 67.7 \\
6 & 191 & 85 & 1.09 & .19 & .98 & -.1 & .99 & .0 & .17 & 62.4 & 61.6 \\
13 & 190 & 85 & 1.05 & .19 & 1.75 & 4.8 & 1.37 & 2.8 & .16 & 62.4 & 61.3 \\
20 & 165 & 85 & .16 & .19 & .99 & .0 & 1.09 & .9 & -.45 & 36.5 & 56.6 \\
\hline MEAN & 185.0 & 85.0 & .90 & .20 & 1.00 & -.1 & .97 & -.1 & & 60.5 & 61.3 \\
S.D. & 17.9 & .0 & .71 & .02 & .29 & 2.2 & .25 & 1.9 & & 13.0 & 5.8 \\
\hline
\end{tabular}

Fig. 5. Person Fit

It goes the same to item fit, from 110 item constructed, 25 item deleted. Again, the suggestion is based on Point Mean Correlation (PTMEA), Mean Square (MNSQ) and z-standard (ZSTD). Item whose MNSQ is nearer to 1 and $z$-std nearer to 0 is deemed a better fit. Remain the same if the item is in between the range of $0.5<\mathrm{MNSQ}<1.5$ and ZSTD \pm 2 logit. Remain the same to this item whose MNSQ is a measure the same but different group of the item to be measured because of content validity is preserved in Figure 6. 


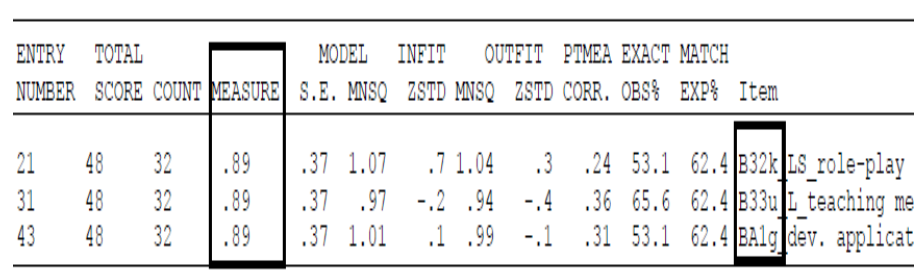

Fig. 6. Consolidated Item Misfit

\section{Unidimensionality}

To ensure the measurement is measuring the specific objective, thus, unidimensionality is crucial. Rasch Analysis applies the Principal Component Analysis (PCA) of the residuals to know on how much variance of the instrument measuring that supposedly to measure. The raw variance explained by measures is $24.8 \%$ closely match the expected $23.7 \%$. However, the analysis shows that only $20 \%$ of unidimensionality requirement minimum. Rasch cut-low point of $40 \%$ is not achieved $[5,19]$. Nevertheless, the unexplained variance in the 1st contrast of good $11.4 \%$ is obtained as tabulated in Figure 7.

\begin{tabular}{|c|c|c|c|}
\hline Total variance in observations & $=$ & $\begin{array}{c}\text { Empirical } \\
113.1 \quad 100.0 \%\end{array}$ & $\begin{array}{r}\text { Modeled } \\
100.0 \%\end{array}$ \\
\hline Variance explained by measures & $=$ & $28.124 .8 \%$ & $23.7 \%$ \\
\hline Unexplained variance (total) & $=$ & $85.0 \quad 75.2 \% 100.0 \%$ & $76.3 \%$ \\
\hline Unexplned variance in 1st contrast & & $12.9 \quad 11.4 \% \quad 15.2 \%$ & \\
\hline
\end{tabular}

Fig. 7. Standardized Residual variance (in Eigenvalue units)

\section{PERSON-ITEM MAP}

\section{A. Item Analysis}

Figure 5 depict the location of the undergraduate practice based on industry personnel experience during their undergraduate study in Requirement Engineering. The location of practice is according to industry personnel ability and difficulty logit. Twenty-nine out of eighty-five items discussed in this paper is referring to identify problem in undergraduate study based on industry personnel experience.

Industry personnel experienced on difficulty which involved seventeen $(0.59 \%)$ items. They are hardly endorsing this item that above ItemMean. Eight items in between ItemMax and PersonMean which can be considered as the most difficult item and seventeen industry personnel not experience those items. Final Examination and Motivation from Lecturer measured inline MeanItem 0.00 logit. This two items show normality of undergraduate Requirement Engineering study. All industry personnel agreed, to easily endorse ten items that below ItemMean.

\section{B. Person Analysis}

There are six female and twenty-one male industry personnel randomly picked from Sharing Services Outsourcing was evaluated. The person-item map shown there is five A's person, eighteen B's person and two C's person. We can generalize that Sharing Services Outsourcing hire more industry personnel from B's person rather than A's and C's person.
The most excellent industry personnel is at highest ability 2.79 logit and seen out of the target. The off target person is the one without corresponding items. The poor industry personnel are at -0.28 logit. The difference between PersonMax and PersonMin is 2.51 logit. The difference is slightly over Standard Deviation (SD) of 0.60 logit. This shows the There are $0.56 \%(\mathrm{~N}=15)$ industry personnel located above MeanPerson and $0.04 \%(\mathrm{~N}=1)$ is below than minimum MeanPerson. In between A's person, located a C's industry personnel above from MeanPerson.

The highest industry personnel at 2.79 logit is female, 23 27 years old, hold a first degree from Software Engineering. Earn CGPA 3.50-3.67 and get requirement grade is in between A- to A. Working as software developer with experience five years and less in software industry. Motivates in successfully finishing project are self-esteem, token from company and responsibility. She involved in 6 to 10 projects in HLE and software industry that involved web-based and multimedia project. She had an experience as developer and system designer.

Other A's person that close to ItemMax is male. His age is 33 and above. Hold Master in Information Technology (IT). He earn CGPA 3.50-3.67 during his first degree with grade Ato $\mathrm{A}$ in Requirement Engineering subject. He had an experience 10 years and above which involved web-based, networking, stand-alone system, others project software development. Experience in doing a software development project is 10 and above project at HLE and software industry. $\mathrm{He}$ had an experience as developer, system engineer, system developer, documenter and project manager.

There is B's person below PersonMin is male, age in between 33 and above. He holds First Degree in Computer science. He manages to get CGPA 2.50-2.99 with grade D- to $\mathrm{D}$ in Requirement Engineering. $\mathrm{He}$ has five and less experience in networking, He had 5 and less experience in HLE and one to five project at industry as a developer.

C's person that range in between A's person is male, age 33 and above, diploma, network, 2.00-2.49,C- to C, system engineer, experience 5 years and less, manufacturing, motivates in successfully finishing project is token from company, rate current learning is average, involved webbased,5 and less project in HLE, 1-5 in working environment, need training after HLE, need tool to capture requirement, average in using internet to finish the project.

Contradict with C's person above PersonMin and B's person is male, age 33 and above, diploma, computer science, 2.00-2.49, C- to C+, software engineer, 5-10 year experience, multimedia project, 5-less project in HLE, 5-10 project experience, experience as developer only.

However, all persons (A's, B's and C's person) agree that they need training after Higher Learning Education (HLE). In increasing the skill among undergraduate students, tool is much recommended to capture requirement from stakeholder. Internet is very useful to finish the project. 


\section{CONCLUSION}

As a conclusion, Rasch help to identify missing data. Person and item misfit is managed with careful manner. Content, construct and predictive validity are maintained. Based on Person-Item Map in Figure 8, the unidimensionality is achieved. Research objective to identify the problem during undergraduate study based on industry personnel is achieved and successfully discussed using Rasch Measurement Model. The industry field required more than CGPA achievement. They are facing the real development project which need a skill worker to handle it. The average CGPA needed by the industry is in range 2.50 and above. The skill worker that have the experience in handling the project more than five development project were very highly recommended to field the position in the software industry.

Future work will be discussed on further analysis based on expert recommendation using QSR NVIVO tool to enhance employability skill for Software Engineering undergraduate in HLE.

\section{REFERENCES}

[1] Al-Qardhawi, Y. (2003). Sunnah: Sumber ilmu dan peradaban. Penterjemah. (M. Firdaus, Ed.) Selangor: International Islamic Thought Malaysia.

[2] Azlinah Mohamed, Azrilah Aziz, Sohaimi Zakaria and Mohd saidfudin Masodi (2008). Appraisal of Course learning Outcomes using Rasch Measurement: A Case Study in Information Technology Education. Conference Proceeding $7^{\text {th }}$ WSEAS International Conference on Artificial Intelligent, Knowledge Engineering and Databases (AIKED '08), 20-22 February, 2008, University Cambridge, Cambridge, UK

[3] Baghaei P. The Rasch Model as a Construct Validation Tool,Rasch Measurement Transactions, 2008, 22:1 p. 1145-6 Chapman, A. (2006). Bloom's Taxonomy - Learning Domains. vol. 2007: Businessballs.com.

[4] Bloom B. S. (1956). Taxonomy of Educational Objectives, Handbook I: The Cognitive Domain. New York: David McKay Co Inc.

[5] Conrad et al. (2011). Validation of the Crime and Violence Scale to the Rasch Measurement Model GAIN Methods Report 1.2.p.10.

[6] Fisher, W. P. J. (2007). Rating scale instrument quality criteria. Rasch Measurement Transactions, 21(1), pg 1095.

[7] Gothwal VK., Wright T.A., (2009). Lamoureux E.L., Pesudovs K, Using Rasch analysis to revisit the validity of the Cataract TyPE Spec instrument for measuring cataract surgery outcomes, Journal of Cataract Refractive Surgery - Vol 35: p.1509-1517.

[8] Hamzah A. G. \& Saidfudin, M. (2009). Modem measurement paradigm in Engineering Education: Easier to read and better analysis using Rasch-based approach. 2009 International Conference on Engineering Education (1CEED 2009), December 7-8, 2009, Kuala Lumpur, Malaysia.

[9] Jamaludin, N. A. A., Sahibuddin, S. \& Hidayat, N. H. (2011). Challenges of Project-Based Learning Towards Requirement Engineering. The 10th WSEAS International Conference on Software Engineering, Parallel and Distributed Systems (SEPADS '11)". University Cambridge, Cambridge, UK.

[10] Jamaludin, N. A. A., Sahibuddin, S. (2011). Development Strategy using Cognitive Domain in e-Requirement Engineering Learning System. IJCSI International Journal of Computer Science Issues, Vol. 8, Issue 5, September 2011. ISSN (Online): 1694-0814. www.IJCSI.org.

[11] Jamaludin, N. A. A., Sahibuddin, S., Jusoff, K., Hidayat, N. H. (2010). Development of a Project-Based Learning Approach in Requirement Engineering. International Journal of Computer Science and Information Security, IJCSIS, Vol. 8, No. 9, pp. 6.

[12] Leedy, P., Ormond, J. (2011). Practical Research:Planning and Design, Pearson.

[13] Linacre J.M. (2001). Category, Step and Threshold: Definitions \& Disordering, Rasch Measurement Transactions, 15:1 p.794.
[14] Linacre, J. M. (2011). Winsteps Rasch Measurement Version 3.71. Winstep.com.

[15] Lopez W (1996) Communication Validity and Rating Scales. Rasch Measurement Transactions 10:1 p. 482-3)

[16] Multimedia Development Corporation (MDeC). http://www.mscmalaysia.my, retrieved $23^{\text {rd }}$ November 2011).

[17] Pohl, M. (2000). Learning to Think, Thinking to Learn: Models and Strategies to Develop a Classroom Culture of Thinking. Cheltenham, Vic.: Hawker Brownlow.

[18] Ramli M. I. (2001). Disclosure in annual reports: an agency theoretic perspective in an International setting. Unpublished Doctoral Dissertation. University of Plymouth, United Kingdom.

[19] Reckase, M.D. (1979). Unifactor Latent Trait Models Applied to Multifactor Tests: Results and Implications, Journal of Educational Statistics, Vol. 4, No. 3, pp. 207-230.

[20] Saidfudin, M., Azlinah M., Azrilah A. A., NorHabibah, A., Hamzah A. G. \& Sohaimi, Z., (2008). Application of Rasch Model in validating the construct of measurement instrument", in International Journal of Education and Information Technologies, Issue 2, Volume 2,. pp. 105112.

[21] Saidfudin, M., Hamza, A. G., Razimah, A. \& Rozeha, A. (2008). Application of Rasch-based ESPEGS Model in Measuring Generic Skills of Engineering Students: A New Paradigm, WSEAS Transactions on Advances in Engineering Education, Issue 8 Vo1.5, WSEAS Press. pp. 591-602, August 2008.

[22] Solemon, B., Sahibuddin, S., Ghani, A.A.A. (2009) Requirements engineering problems and practices in software companies: An industrial survey. International Conference on Advanced Software Engineering and Its Applications, ASEA 2009 Held As Part of the Future Generation Information Technology Conference, pp. 70-77. FGIT 2009, Jeju Island, Korea, December 10-12.

[23] Wright B. D. and Mok, M. M. C. (2004). An overview of the family of rasch measurement models," in Introduction to Rasch Measurement: Theory, Models, and Applications, J. Everett V.Smith and R. M.Smith, Eds. p. 979.

[24] http://www.uky.edu/ kdbrad2/AERA2005SupplyDemandPaper.pdf;

[25] http://www.wseas.us/e-library/conferences/2010/Penang/MMF/MMF25.pdf;

[26] http://www.docstoc.com/docs/55062758/Rasch-Workshop-Booklet--Structu;

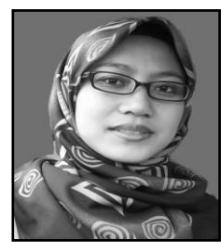

\section{AUTHORS PROFILE}

Nor Azliana Akmal Jamaludin is a Lecturer for Bachelor of Science Software Engineering and Head of Developer for Master Degree Software Engineering program at Universiti Industri Selangor. She received the Master Degree in Computer Science (Real-Time Software Engineering) from Advanced Informatics School (formerly known as Centre for Advanced Software Engineering (CASE), Universiti Teknologi Malaysia, in 2004. Currently, she is doing her Doctorate in Computer Science, specialize in Software Engineering at UTM and supervise by Prof. Dr. Shamsul Sahibuddin. She is a member of Malaysian Software Engineering Interest Group, Malaysia. Her field of expertise is in software requirement, analysis, system integration, and elearning. Her current research interest is on techniques that can enhance skill among Software Engineering undergraduate of higher institutions in Malaysia using machine learning system.

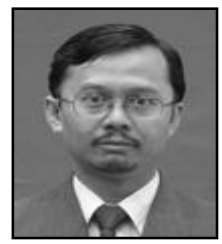

Shamsul Sahibuddin received the $\mathrm{PhD}$ in Computer Science at Aston University, Birmingham, United Kingdom in 1998 and Master Science (Computer Science) at Central Michigan University, Mt. Pleasant, Michigan in 1988. He is currently a Dean / Professor at Advanced Informatics School, Universiti Teknologi Malaysia (UTM) and has been a Member of the Program Committee for Asia-Pacific Conference on Software Engineering. His field of expertise is in software requirement, software modelling technique, software development and software process improvement. He has been very active in scholarly journals-book writing and publishing. 


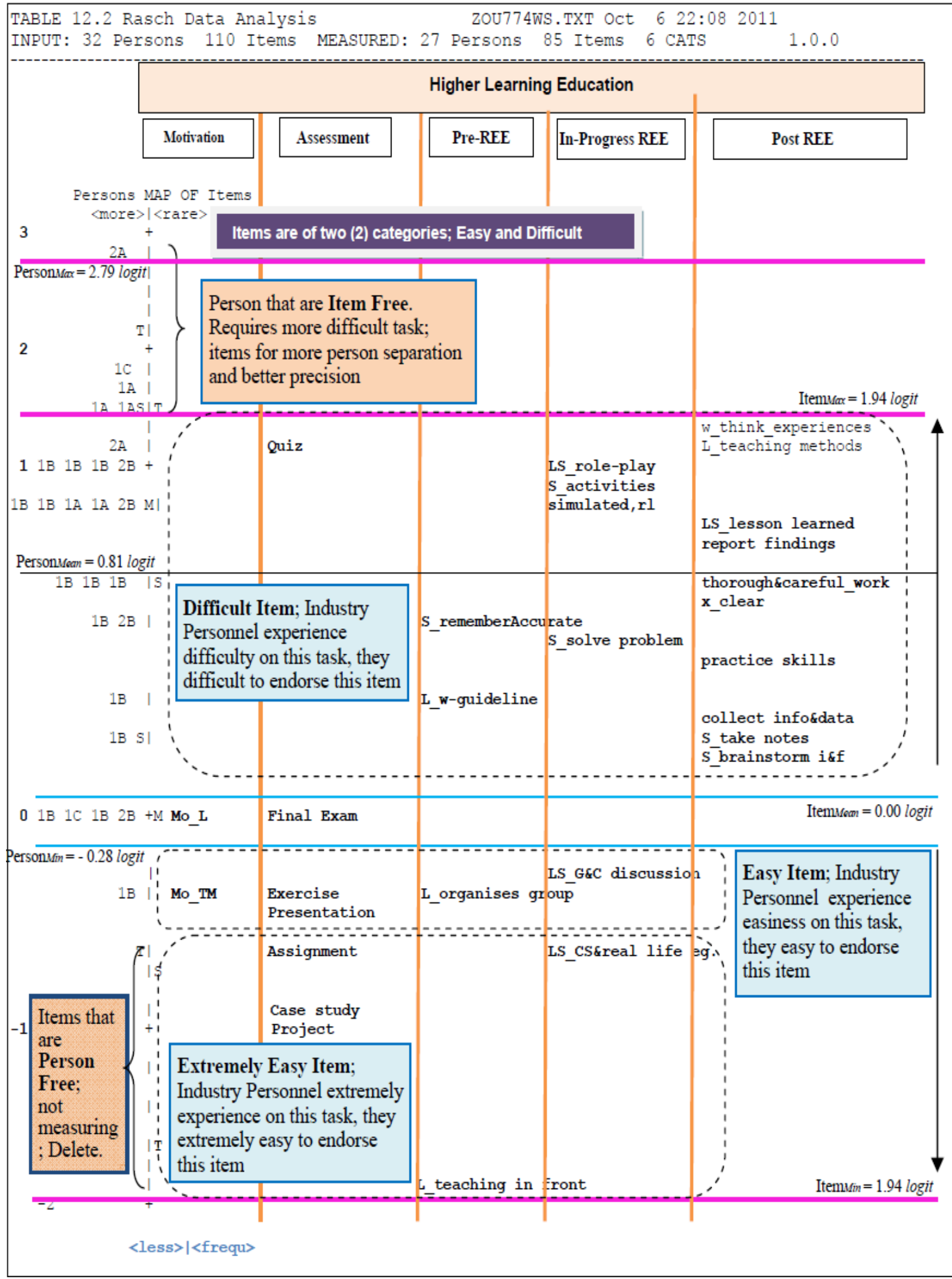

Fig. 8. Person-Item Map for Requirement Engineering Education (REE) 